\title{
Cancer driver candidate genes AVL9, DENND5A and NUPL1 contribute to MDCK cystogenesis
}

\author{
Yaping $\mathrm{Li}^{1}{ }^{1}$, , Jianing $\mathrm{Xu}^{1,4, *}$, Huan Xiong ${ }^{1}$, Zhongyao Ma ${ }^{1}$, Zhenghe Wang ${ }^{2}$, Edward \\ T. Kipreos ${ }^{3}$, Stephen Dalton ${ }^{1}$ and Shaying Zhao ${ }^{1}$ \\ ${ }^{1}$ Department of Biochemistry and Molecular Biology, Institute of Bioinformatics, University of Georgia, Athens \\ ${ }^{2}$ Department of Genetics \& Genome Sciences and Case Comprehensive Cancer Center, Case Western Reserve University, \\ Cleveland, Ohio \\ ${ }^{3}$ Department of Cellular Biology, University of Georgia, Athens \\ ${ }^{4}$ Current Address: Human Oncology \& Pathogenesis Program, Memorial Sloan-Kettering Cancer Center, New York \\ * These authors contributed equally to this work
}

Correspondence to: Shaying Zhao, email: szhao@bmb.uga.edu

Keywords: AVL9; DENND5A; NUPL1; MDCK; Cytogenesis; Apicobasal Polarity

Received: November 10,2014 Accepted: December 14, $2014 \quad$ Published: December 15, 2014

This is an open-access article distributed under the terms of the Creative Commons Attribution License, which permits unrestricted use, distribution, and reproduction in any medium, provided the original author and source are credited.

\section{ABSTRACT}

AVL9, DENND5A and NUPL1 are among the cancer driver candidate genes previously identified via dog-human comparison, and may function in epithelial cell polarity as indicated by bioinformatics analysis. To better understand their cellular functions and roles in cancer, we knocked down each gene in MDCKII cells through shRNA and performed three-dimensional culture. Compared to the control, the knockdown clones developed significantly more abnormal cysts, e.g., cysts with the lumen harboring dead and/or live cells, or cysts having multiple lumens. Further analysis revealed that abnormalities initiated at the first cell division and persisted throughout the entire cystogenesis process. For NUPL1-knockdown cells, abnormal cytogenesis largely arose from faulty cell divisions, notably monopolar spindles or spindles with poorly separated poles. For AVL9- or DENND5A-knockdown cells, abnormalities originated from both aberrant intracellular trafficking and defective mitosis. Moreover, while all knockdown clones displayed an accelerated rate of both cell proliferation and death, only AVL9- and DENND5A-knockdowns, but not NUPL1-knockdown, promoted cell migration. These observations indicate that NUPL1 contributes to bipolar spindle formation, whereas AVL9 and DENND5A participate in both intracellular trafficking and cell cycle progression. Our study shed lights on these genes' normal cellular functions and on how their alteration contributes to carcinogenesis.

\section{INTRODUCTION}

Via dog-human comparative genomics and oncology studies, we have identified 73 driver candidate genes (likely cancer-causative when altered) and 38 passenger candidate genes (of which alterations are unlikely cancercausative) for colorectal cancer [1-3]. Bioinformatic analysis indicates that whereas passenger candidate genes' functions appear to be random, driver candidate genes are significantly enriched in functions that are associated with epithelial apicobasal polarity establishment and maintenance [1]. This is consistent with the observation that loss of cell polarity is a hallmark of epithelial cancers $[4,5]$, and supports the notion that epithelial polarity is a tumor suppressor [4-11].

AVL9, DENND5A and NUPL1 are among the 73 driver candidate genes described above. They are deleted in colorectal cancer and restrict cell proliferation of HCT116 and other cancer lines [1]. They are hence classified as putative tumor suppressors. Furthermore, the three genes are annotated to participate in intracellular trafficking that may relate to epithelial cell polarity establishment and maintenance. NUPL1 encodes nucleoporins p58 and p45 which are components of 
the nuclear pore complex (NPC) [12], a large transport channel regulating molecular trafficking across the nuclear membrane. DENND5A contains DENN (differentially expressed in neoplastic versus normal cells) domains and interacts with Rab11, Rab6 and Rab39, small GTPases that are indispensable to intracellular membrane trafficking [13-15]. AVL9, having DENN-related AH (Av19 homology) domains, is an exocytosis gene in yeast [16] and is involved in cell migration [17]. Other than these, no other published studies indicate the involvement of the three genes in cancer or cell polarity.

To experimentally determine if AVL9, DENND5A and NUPL1 function in epithelial cell polarity and to better understand how they restrict cancer cell proliferation, we set out to knock down each gene in MDCKII (MadinDarby canine kidney II) cells, a well-established cell line model for studying cell polarity and epithelial morphogenesis [18-20], and to examine the effects of the gene knockdown on the MDCKII cystogenesis via three dimensional (3D) culture [21].

\section{RESULTS}

\section{$A V L 9, D E N N D 5 A$ and $N U P L 1$ are knocked down in MDCKII cells through shRNA}

Using different shRNA constructs targeting various regions of the AVL9 gene (Supplementary Table S1), we generated four independent MDCKII clones with stable AVL9-knockdown. Meanwhile, we also established two independent MDCKII clones with stable knockdown for each of the DENND5A and NUPL1 genes. Compared to the control, the mRNA reduction rate in the knockdown clones is $70-90 \%$ for $A V L 9, \sim 50-60 \%$ for DENND5A
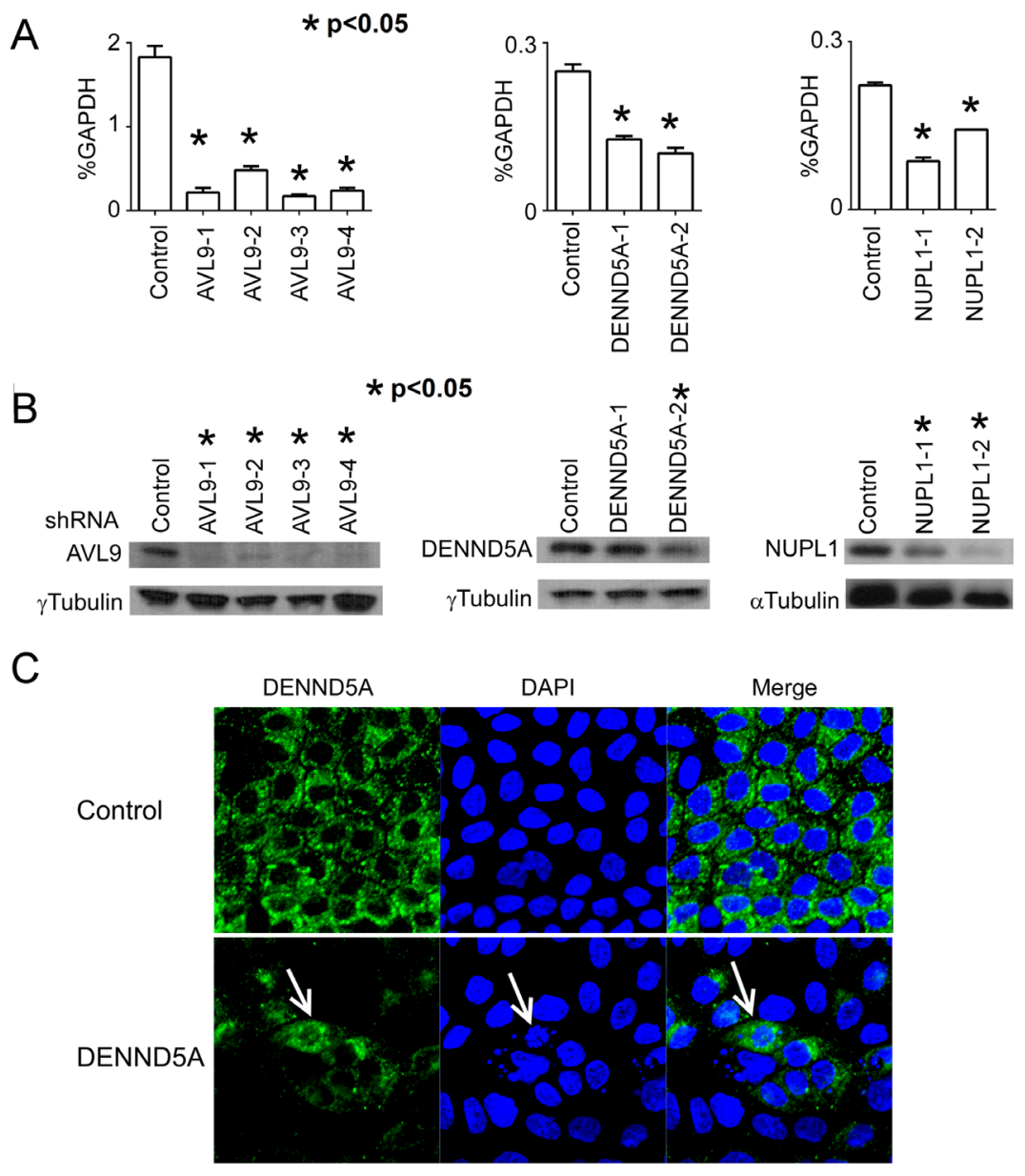

Figure 1: $A V L$ 9, DENND5A and NUPL1 are knocked down in MDCKII cells via shRNA. The control clone is with shRNA against the lacZ gene. The four $A V L 9$-knockdown clones are labeled as $A V L 9-1,-2,-3$ and -4. Similarly labeled are also the two $D E N N D 5 A$ knockdown clones and the two NUPL1-knockdown clones. (A and B) The mRNA (A) and protein (B) expression levels of $A V L 9$, DENND5A and NUPL1 in each clone were quantified by qRT-PCR and western blot, respectively. The p-values represent the difference in the target gene expression between a knockdown clone and the control, calculated by t-tests with at least three biological replicates. (C) Representative immunostaining images with the anti-DENND5A antibody showing that some abnormal cells (e.g., with fragmentation of the nucleus as pointed by the arrows) have very strong DENND5A expression. This explains why the protein depletion level determined by western blot of $D E N N D 5 A$-knockdown clones is insignificant or not as significant as other gene-knockdown clones (B). 
and $30-60 \%$ for NUPL1 (Figure 1A). At the protein level, the depletion rate is near $90 \%$ for AVL9, $>50 \%$ for NUPL1 and $<50 \%$ for DENND5A (Figure 1B). Based on our analysis, the lower DENND5A protein depletion rates as determined by western blot experiments do not reflect a failed gene-knockdown, but rather are caused by significantly increased protein expression in certain abnormal/apoptotic cells that emerged because of DENND5A-knockdown (Figure 1C). Lastly, the knockdown is well-maintained in each clone, being consistent after 9 cell passages (Supplementary Figure S1). All experiments described below were performed with passage 5 cells.

\section{$A V L 9, D E N N D 5 A$ or NUPL1 knockdown all alter MDCKII cyst structure}

To test the hypothesis that each of the three genes plays a role in epithelial morphogenesis as indicated by bioinformatics analysis [1], we took advantage of MDCKII cells' ability to form cysts with apicobasal polarity in $3 \mathrm{D}$ culture. Our results show that in our $3 \mathrm{D}$ culture environment, $>90 \%$ of the control cells formed well-organized and approximately equal-sized cysts (Figures 2A, 2E and 2F). Each cyst has a single, cell- or debris-free, and clearly-defined lumen surrounded by a monolayer of epithelia that consist of cells with wellestablished apicobasal polarity (Figure 2A). This is illustrated with E-cadherin, a cell-cell adherens junction marker that stains the lateral membrane, and F-actin, which nicely outlines the apical region and the lumen (Figure 2A). These well-organized cysts are referred to as "single-lumen cysts" hereafter.

In contrast, AVL9-, DENND5A- or NUPL1knockdown cells developed mostly aberrant cysts, which could largely be classified into three types: filled-lumen, small-sized, and multi-lumen (Figures 2B-2E). The "filledlumen cysts" have a single lumen per cyst, but inside the lumen often harbor apoptotic cells (Figure 2G), nuclear fragments and other cellular debris (Figure 2D), and for those of AVL9-knockdown clones, sometimes live cells as well (Figure 2B). The "small-sized cysts" are a subset of "filled-lumen cysts" that are notably smaller in size, with the epithelia of each cyst consisting of significantly fewer but larger and often multinuclear cells (Figures 2B-2D). The "multi-lumen cysts" by definition harbor two or more lumens per cyst (Figures 2B and 2C).
A
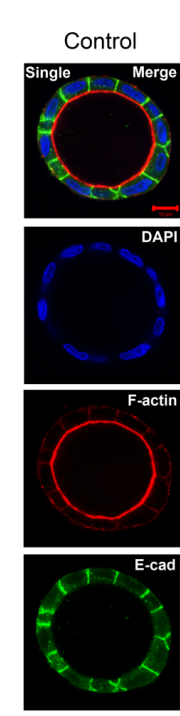

G

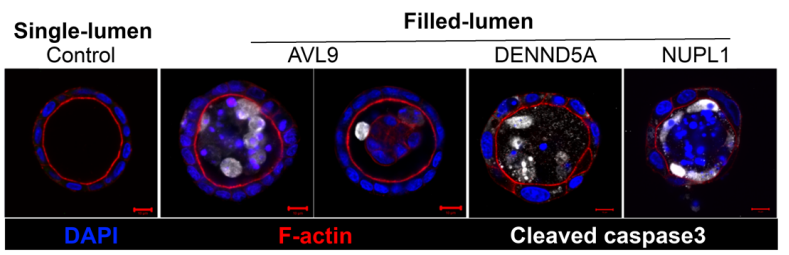

B
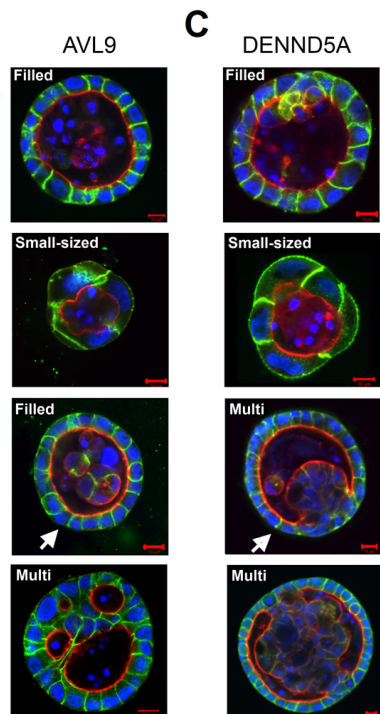

D
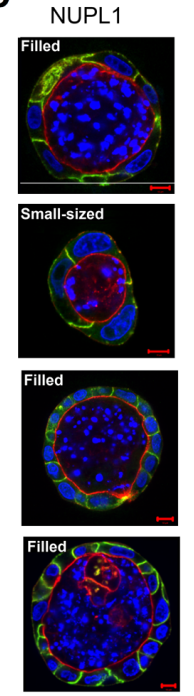

E

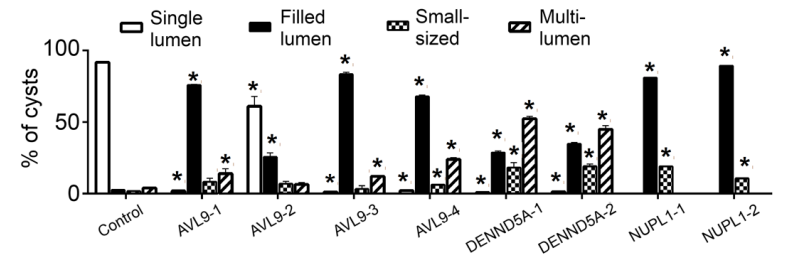

$\mathbf{F}$
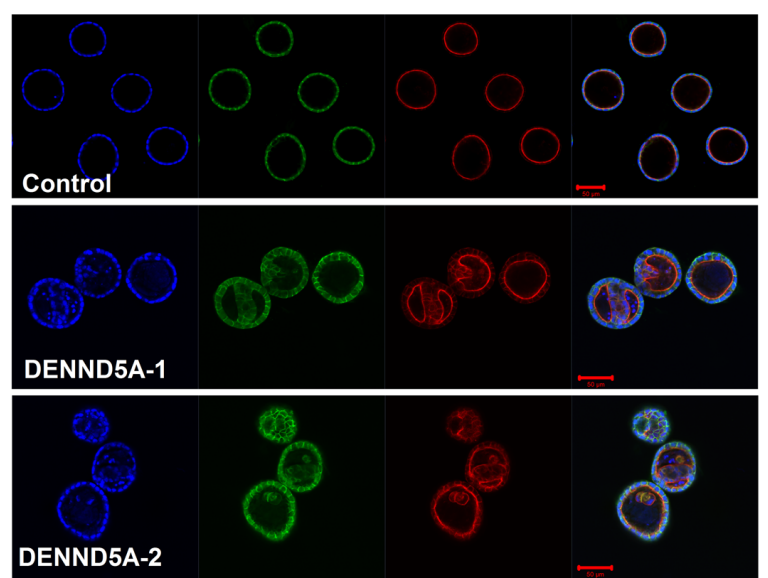

Figure 2: Each gene-knockdown alters cyst structures. (A-D) Confocal images of representative cysts of the control (A), and AVL9-, DENND5A- or NUPL1-knockdown clones (B-D) stained for the nucleolus (DAPI), F-actin, and E-cadherin (E-cad). The images represent major cyst types including single-lumen, filled-lumen, small-sized, and multi-lumen. The white arrow in point to the cyst that harbors live cells in its lumen (B), or the cyst that has cells growing into its lumen forming the polyp-like phenotype (C). (E) Percentage of the cyst types illustrated in (A-D) summarized from >100 cysts examined for each clone. Error bars were calculated with three biological repeats. The p-values indicate the difference in the total cyst number of each type between a knockdown clone and the control, calculated by t-tests. (F) An example of confocal images used to compute the data shown in (E). (G) Filled-lumen cysts harbor apoptotic cells in their lumen, as indicated by cleaved caspase3- staining. 
NUPL1-knockdown leads to predominantly filled-lumen cysts, while DENND5A- or $A V L 9$ knockdown results in both filled-lumen and multilumen cysts

The distribution of filled-lumen cysts, small-sized cysts, and multi-lumen cysts varies among the geneknockdowns (Figure 2E). For NUPL1-knockdown clones, nearly all cysts are filled-lumen (Supplementary Figure S2), with the lumen harboring apoptotic cells, nuclear fragments and other cellular debris, and with no live cells observed (Figures 2D, 2E and 2G). Other than being filledlumen, these cysts however appear to retain apicobasal polarity. Indeed, surrounding the lumen is a single layer of cells with F-actin primarily located at the apical side and E-cadherin mostly concentrated at the lateral or sometime basolateral membrane, although F-actin stained more weakly when compared to the control cysts (Figures $2 \mathrm{~A}$ and 2B). NUPL1-knockdown also yielded small-sized cysts, but not multi-lumen cysts (Figure 2E).

Filled-lumen cysts are also frequent for both AVL9and $D E N N D 5 A$-knockdown clones. However, a phenotype unique only to $A V L 9$-knockdown is that some of its filledlumen cysts harbor live cells, which even formed cell mass with lumen-facing apical polarity (see the white arrow-pointed cyst in Figure 2B). Another difference is that $A V L 9$-knockdown clones developed fewer small-sized cysts, compared to the other two genes (Figure 2E).

Differing from NUPL1-knockdown, both $D E N N D 5 A$ - and $A V L 9$-knockdown led to the development of multi-lumen cysts (Figures 2B and 2C). Unlike those
A
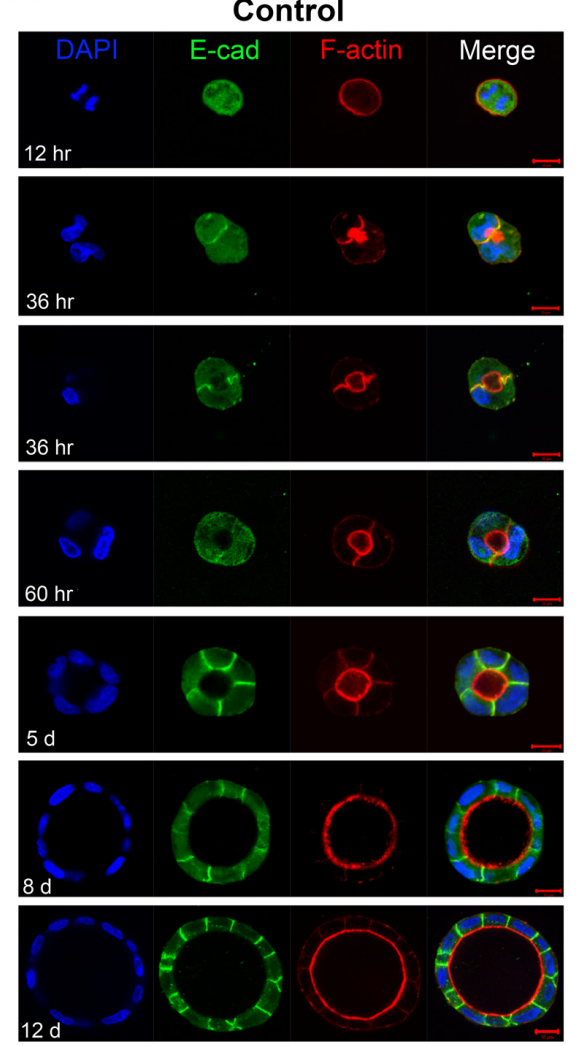

B

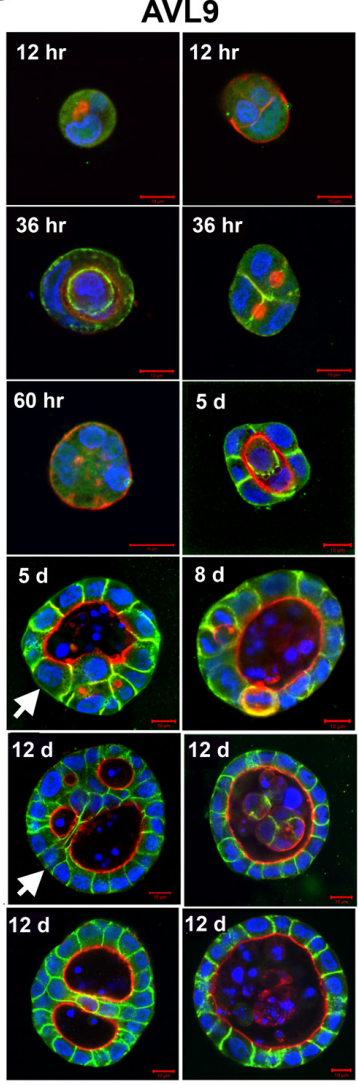

C

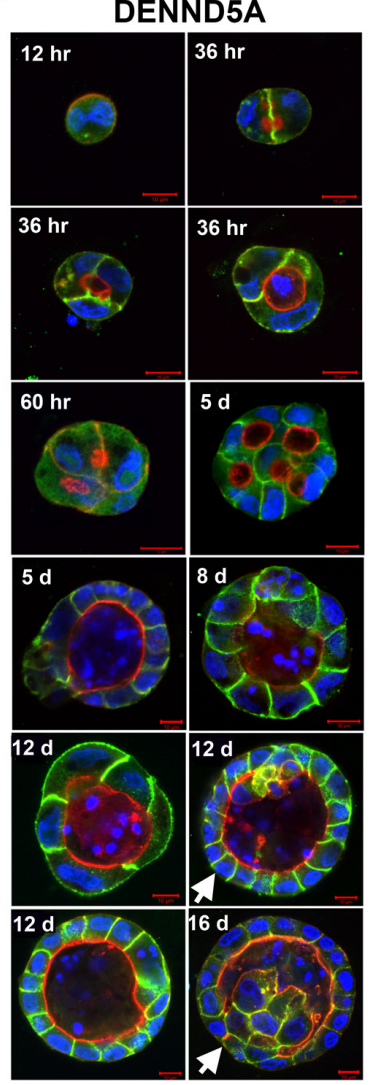

D

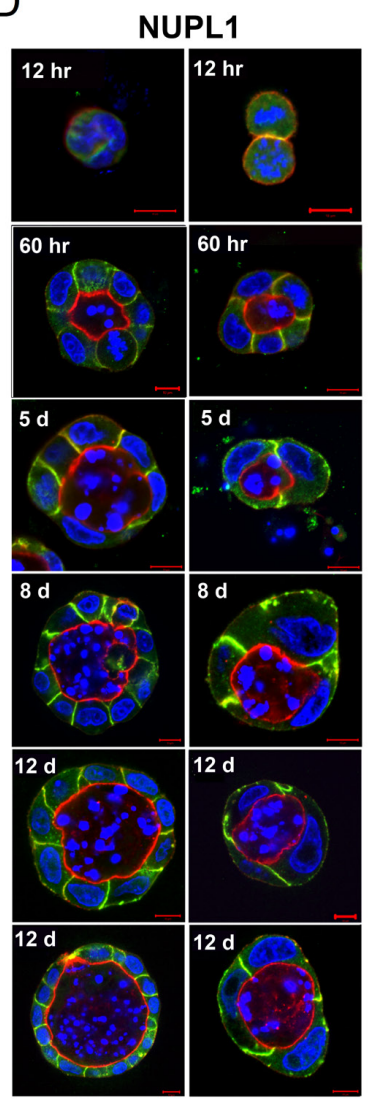

Figure 3: Abnormalities in target gene knockdown clones start at the first cell division and persist throughout the entire cystogenesis process. (A) The control clone's time course of cystogenesis. Representative images shows that lumen formation initiates via polarized intracellular trafficking, as indicated by of F-actin and E-cadherin staining, during the first cell division and completes as early as the two- or three-cell stage (also see Supplementary Figure S3). This period is defined as the lumen-establishing stage. Then, the cyst grows in size through orientated cell division (see Figure 4A), and this period is defined as the lumen-enlarging stage. The time course is indicated by hours (hr) or days (d) after the cell-seeding, e.g., "12 hr" and " 5 d" representing 12 hours and 5 days after seeding respectively. (B and C) The cystogenesis time course of AVL9- or DENND5A-knockdown clones. Representative images show abnormalities at the lumen-establishing stage, including no visible lumen-establishment even at the $>3$ cell stage (the "60 hr" cyst in B), multiple lumen-formation in a single cyst (the " 36 hr" cyst in B; the "36 hr" and "60 hr" cysts in C), and off-centered lumen (the left "36 hr" cyst in C). Abnormalities continued at the lumen-enlarging stage, as shown by de novo lumenogenesis (the " $5 \mathrm{~d}$ " and " $12 \mathrm{~d}$ ' cysts pointed by while arrows in B) and cells actively dividing into the lumen (the "12 d" and " $16 \mathrm{~d}$ " cysts pointed by white arrows in C). (D) The cystogenesis time course of NUPL1-knockdown clone. Note that throughout the cystogenesis, filled-lumen development was widespread but no multi-lumen cysts were observed. 
in filled-lumen cysts, the cells in certain parts of multilumen cysts grew into multiple layers and many cells completely lost their apicobasal polarity (Figures 2B and $2 \mathrm{C}$ ). Interestingly, multi-lumen cysts appear to have arisen from different mechanisms between the two geneknockdowns, and we will examine this difference in the next section.

In control cells, the lumen initiates during the first cell division and expands via polarized cell division

To better understand spatial-temporal regulation of cystogenesis, we imaged cyst development at different time points. For control cells, cell polarity and lumen formation initiated during cytokinesis of the first cell division (Figure 3A). This was shown by polarized trafficking of F-actin and E-cadherin to the newly established junction between the two daughter cells (Figure 3A). F-actin then congregated around the center of the junction that further developed into the lumen, whereas E-cadherin concentrated along the cell-cell junction that further developed into the lateral plasma membrane compartments (Figure 3A). As early as the twoor three-cell stage, the lumen was established (Figure 3A; Supplementary Figure S3). By day five, nearly all cysts exhibited a single well-defined lumen surrounded by an epithelial monolayer (Figure 3A). Once the lumen was established, the structure of a cyst was maintained and grown through oriented cell division, with the spindle aligned at an approximately $90^{\circ}$ angle with respect to the radius of the sphere [22] (Figure 4A). Thus, for the control cells, cystogenesis can be largely divided into three phases: 1) the lumen-establishing stage (seeding to the two or three cell stage; see Figure 3A); 2) the lumen-enlarging stage through orientated cell divisions (primarily from day 3 to day 12; see Figure 3A); and 3) the cyst maintenance stage where most cells cease to divide (after 12 days; not shown in Figure 3A).

\section{In knockdown cells, abnormalities emerge during the first cell division and persist throughout the entire cystogenesis process}

For all knockdown clones, abnormalities start as early as the first cell division and persisted throughout the entire cystogenesis process, affecting both lumen establishment and enlargement as shown in Figures 3B-3D. As elaborated below, abnormalities in cell divisions were observed in all knockdown clones, and in DENND5A- and AVL9-knockdown clones, deregulated intracellular trafficking were also detected.

Defects in both intracellular trafficking and mitosis were observed in AVL9- or DENND5A-knockdown clones. AVL9- or DENND5A-knockdown cells harbor a variety of aberrations. In these cells, abnormal lumenogenesis was evident at the lumen-establishing stage, as shown by no visible lumen-establishment even at the $>3$-cell stage, multiple lumen-formation in a single cyst, or off-centered lumen (Figures $3 \mathrm{~B}$ and $3 \mathrm{C})$. It appears that polarized intracellular trafficking of F-actin and other molecules, essential for lumen-initiation (Figure 3A), was disrupted. At the lumen-enlarging stage, additional de novo lumenogenesis occurred, resulting in the development of multi-lumen cysts. This is especially common in $A V L 9$-knockdown clones (see cysts pointed by white arrows in Figure 3B).

Mitotic defects were also frequently observed in both AVL9- and DENND5A-knockdown clones. At the lumen-establishing stage, these include failed cytokinesis as shown by multi-nucleated cells, abnormal spindles such as mono- or multi-polar spindles, and disorganized microtubules (Figures 4B, 4C and 4E). These abnormalities continued after the lumen was established. Moreover, misaligned spindles emerged at this phase, with the spindle angles significantly diverging from the normal $\sim 90$ degree (Figures 4B, 4C and 4F). Notably, some of these misaligned spindles were otherwise functional and resulted in successful cell division. As a consequence, cells were actively grown into the lumen (see the cysts pointed by the white arrows in Figures $2 \mathrm{C}$ and $3 \mathrm{C}$ ), sometimes yielding multi-lumen cysts (Figures $2 \mathrm{C}$ and $2 \mathrm{~F}$ ). This phenomenon was mostly observed in $D E N N D 5 A$-knockdown clones.

Monopolar spindles or spindles with poorly separated poles were frequently seen in NUPL1knockdown cells

The most notable aberration detected in NUPL1knockdown clones in both 3D cytogenesis and 2D culture is monopolar spindles or spindles with poorly separated poles (Figure 4D). This occurred in 60\% of spindles examined, significantly higher than other gene-knockdown clones (Figure 4E). Interesting, $\gamma$-tubulin staining revealed two centrosomes for many such spindles investigated (Figure 4G). Besides poorly separated spindle poles, a smaller percentage of multipolar spindles and misaligned spindles were also observed (Figures 4E and 4F). However, unlike DENND5A- or AVL9- knockdown clones, we did not evidently perceive deregulated intracellular trafficking in NUPL1-knockdown clones.

\section{AVL9 and DENND5A, but not NUPL1, knockdown promote cell migration}

Compared to the control, all knockdown clones exhibited accelerated cell proliferation accompanied by increased cell death (Figure 3), consistent with previous studies [1]. We further tested whether knockdown of each 
gene would alter cell migration, another important feature of cancer. We carried out scratch wound healing assays and found that the cell migration rate differed among these gene-knockdowns. While AVL9- and DENND5Aknockdown clones all migrated significantly faster throughout the time course of the assay than the control cells (Figures 5A and 5B), NUPL1-knockdown clones did not (Figure 5C). The finding supports that AVL9 and $D E N N D 5 A$, but not $N U P L 1$, play a role in cell migration. It is consistent with the observation that cell-cell adherens junctions, as indicated by E-cadherin staining (Figure 5D), are better preserved in NUPL1-knockdown cells than in AVL9- or DENND5A-knockdown cells (Figure 5D).

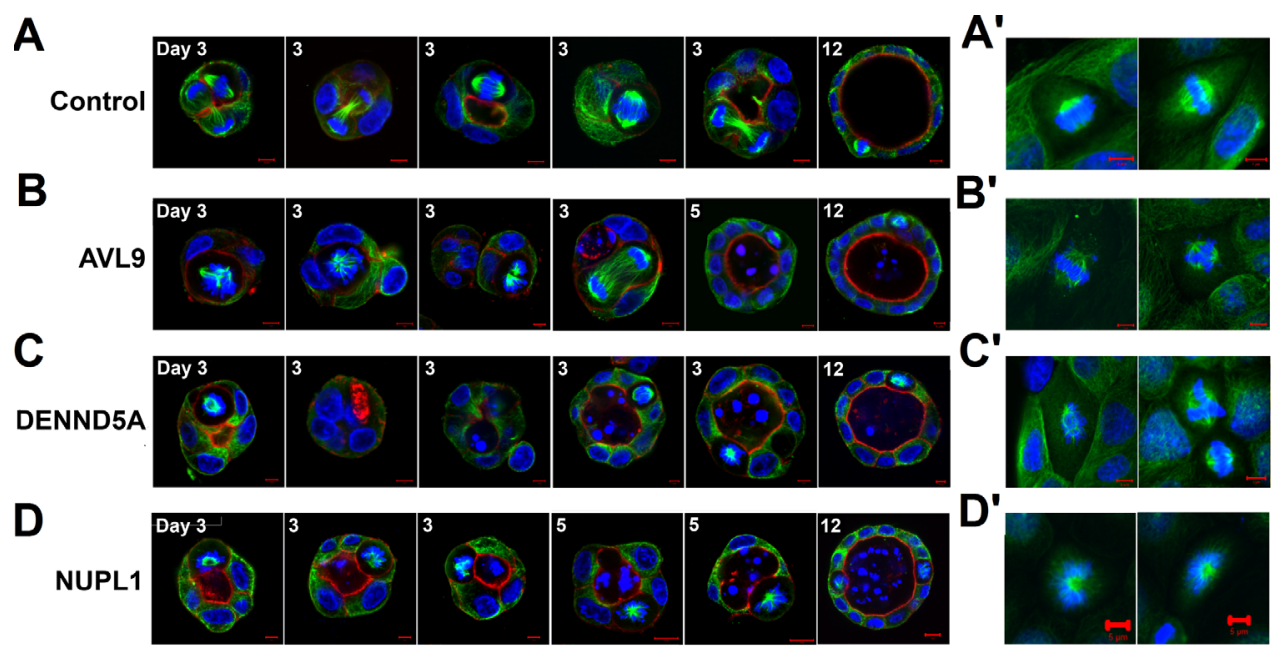

E

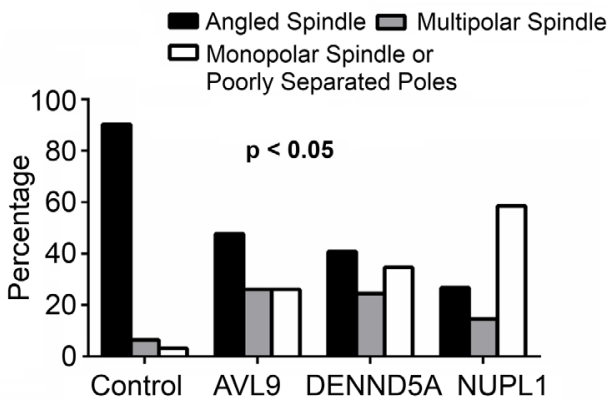

G

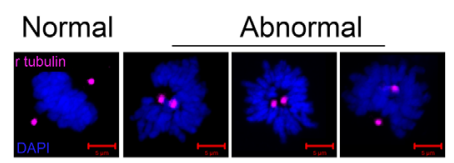

$\mathbf{F}$

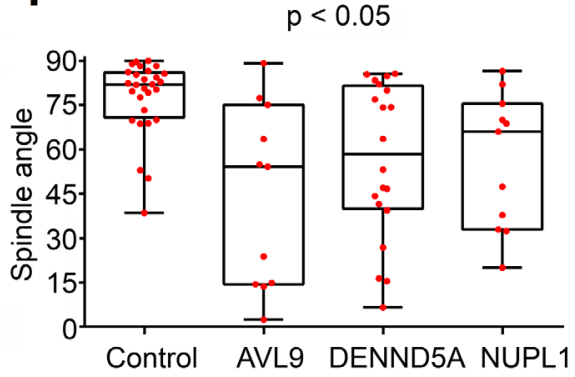

G' ${ }^{100}$

Figure 4: Target gene knockdown clones develop abnormal spindles and other defects. (A and A') Control cells' spindles in 3D (A) and 2D (A') culture. Representative images show correctly-oriented spindles and well-organized cell division at both early (day 3) and late (day 12) stages of cystogenesis in control cells. Images of "Day 3" present cell division views from various angles (top, side, center, etc.) of the cyst. (B and B') AVL9-knockdown cells developed spindles in 3D culture (B) that are multipolar ( $1^{\text {st }}$ image), monopolar or with poorly separated poles ( $2^{\text {nd }}$ and $3^{\text {rd }}$ images $)$, with disorganized microtubules ( $4^{\text {th }}$ image), or misoriented $\left(5^{\text {th }}\right.$ and $6^{\text {th }}$ images). Multipolar spindles were observed in 2D culture (B'). (C and C') DENND5A-knockdown cells in 3D (C) and 2D (C') culture developed similar spindle abnormalities as in (B and B'), along with defects in cytokinesis indicated by the multinucleated in the $2^{\text {nd }}$ image in (C). (D and D') NUPL1-knockdown cells developed many monopolar spindles or spindles with poorly separated poles $\left(1^{\text {st }}\right.$ and $4^{\text {th }}-6^{\text {th }}$ images$)$ (see also Supplementary Figure S4), along with other abnormalities such as multipolar spindles ( $2^{\text {nd }}$ and $3^{\text {rd }}$ images) in 3D culture (D). Spindles with poorly separated poles were also frequently observed in 2D culture (D'). (E) Statistic summary of different types of abnormal spindles shown in (A-D), compiled from $>50$ cysts examined for each clone. (F) Spindle orientation for those "Angled Spindles" shown in (E) of the control and target gene knockdown clones. The spindle orientation is indicated by the spindle angle with respect to the radius of the cyst sphere, as described previously [22]. Error bars represent the standard error of the mean. (G and G') $\gamma$-tubulin-staining indicates two centrosomes in monopolar spindles or spindles with poorly separated poles developed by NUPL1-knockdown cells shown in (D and D'). Representative images of normal and abnormal 2D metaphase cells are shown in (G), and the statistics from at least 30 metaphase cells examined for each clone are shown in (G'). The p-values (all below 0.05) in panels E, F and G' indicate the difference in spindle types (E), angles $(\mathrm{F})$ and kinds $\left(\mathrm{G}^{\prime}\right)$ as shown between a specified knockdown clone and the control by t-tests. 


\section{DISCUSSION}

Epithelial cells are polarized, with distinct apical and basolateral membrane domain and directional intracellular trafficking (e.g., from ER to Golgi, to endosomes and to plasma membrane). Epithelial morphogenesis requires precise regulation of both cell division and polarity establishment. During carcinogenesis, cell division is deregulated, cell polarity is lost, and epithelial tissue architecture is broken down. We report here that three genes, AVL9, DENND5A and NUPL1, which are recurrently deleted in colorectal cancer [1], all contribute to epithelial morphogenesis.

\section{NUPL1 contributes to epithelial morphogenesis by promoting bipolar spindle formation}

NUPL1 encodes nucleoporins p58 and $\mathrm{p} 45$, components of the p62 complex that makes up the central channel of the nucleopore, regulating molecular trafficking across the nuclear membrane [12, 23]. During mitosis when the nuclear membrane is disintegrated, NUPL1 however plays a different role - promoting bipolar spindle assembly, as revealed by our study. NUPL1-knockdown leads to the phenotype of monopolar spindles or spindles with poorly separated poles in both 3D and 2D MDCKII cell culture. These abnormal spindles often result in cell cycle arrest and apoptosis $[24,25]$. Indeed, throughout the cytogenesis of NUPL1-knockdown clones, apoptotic cells have been frequently observed being ejected into the lumen, leading to the widespread development of filledlumen cysts.

While neither nucleoporin p58 nor p45 has been reported to be involved in cell cycle regulation, other nucleoporins are known to contribute to successful mitosis [26]. Significantly, depletion of Nup98 also leads to monopolar spindle development [27]. The authors have further proposed that Nup98 regulates microtubule dynamics by antagonizing the activity of MCAK (microtubule-depolymerizing mitotic centromereassociated kinesin) to assist bipolar spindle assembly. Whether NUPL1 also functions through a similar mechanism remains to be determined. Besides deregulated
A

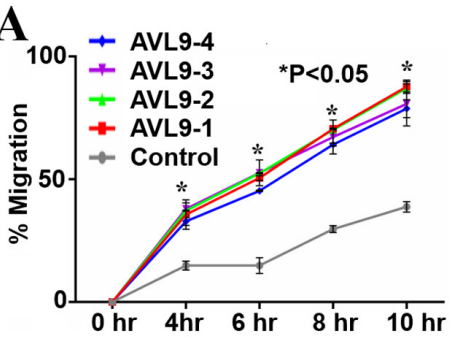

B

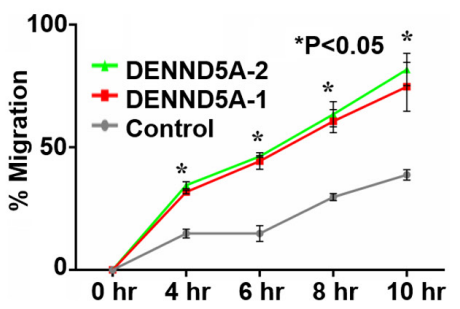

$\mathrm{C}$

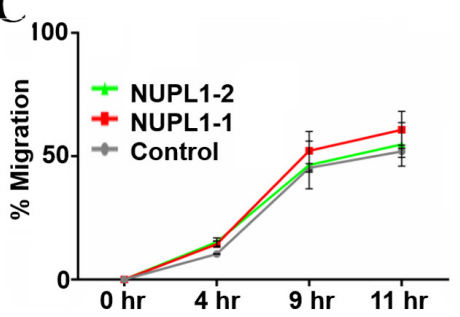

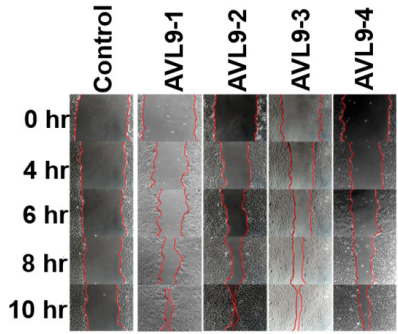
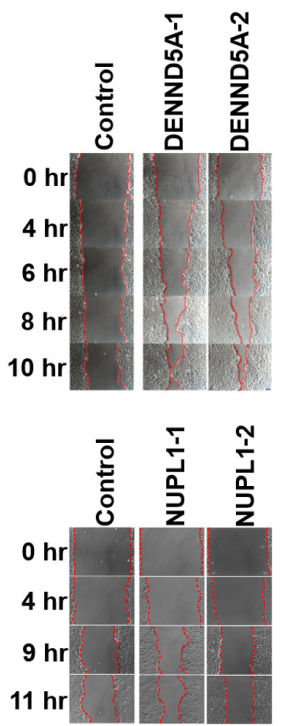
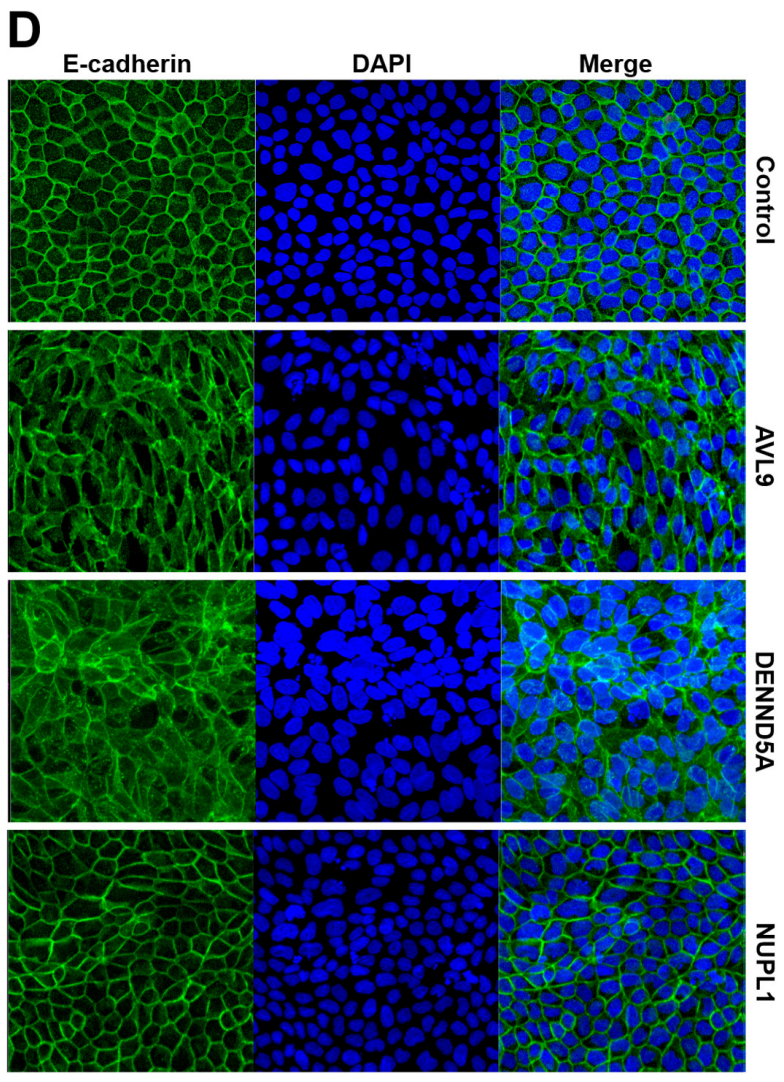

Figure 5: $A V L$ 9- and $D E N N D 5 A$-knockdown, but not NUPL1-knockdown, promotes MDKCII migration. (A-C) Time course experiments showing the difference in cell migration between the control and the knockdown clones of $A V L 9$ (A), DENND5A (B), or NUPL1 (C). The plots on the left indicate the cell immigration results at each time point summarized from three biological repeats, one of which is shown in images on the right. The p-value indicate the difference in cell migration between a knockdown clone and the control at an indicated time point, calculated by t-tests from three biological replicates. (D) E-cadherin and DAPI staining of 2D cell culture of the control and target gene knockdown clones as indicated. As revealed by E-cadherin staining, the cell-cell adherens junctions are disrupted in $A V L 9$ - or DENND5A-knockdown cells but are largely preserved in NUPL1-knockdown cells, when compared to the control cells. 
microtubule dynamics, monopolar spindles can also arise from failed centrosome duplication or separation [24]. This mechanism is, however, unlikely in NUPL1knockdown cells, because most of the monopolar spindles do have two centrosomes.

While NUPL1-knockdown reduces the apicobasal polarity of the plasma membrane somewhat, it does not completely abolish it. Notably, the cell-cell adherens junctions are largely preserved and NUPL1-knockdown has no effects on cell migration. Hence, NUPL1 is unlikely to be involved in intracellular trafficking that targets adhesion proteins to the plasma membrane, differing from DENND5A or AVL9 described below.
$D E N N D 5 A$ and $A V L 9$ contribute to epithelial morphogenesis by regulating intracellular trafficking and cell cycle progression

In addition to mitotic aberrations such as failed cytokinesis and abnormal spindles, the knockdown of $A V L 9$ or DENND5A has also led to altered intracellular trafficking, disrupted cell-cell adherens junctions and faster cell migration, which have not been observed in NUPL1-knockdown cells. Unlike NUPL1, both AVL9 and DENND5A are cytoplasmic proteins. Importantly, DENND5A and possibly AVL9 are regulators of Rab GTPases [15, 17]. DENND5A, also called Rab6IP1 (Rab6-interacting protein 1) in mice, binds Rab6 and

A
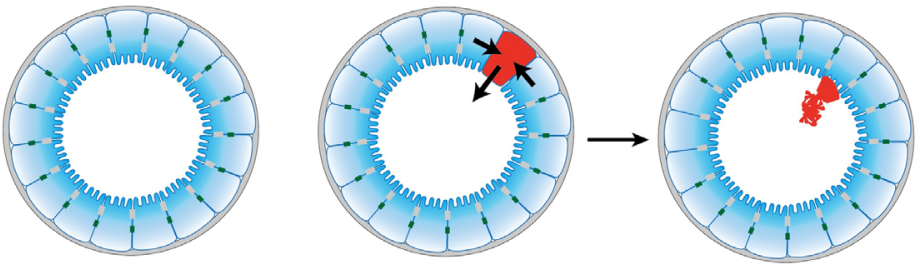

Normal

B
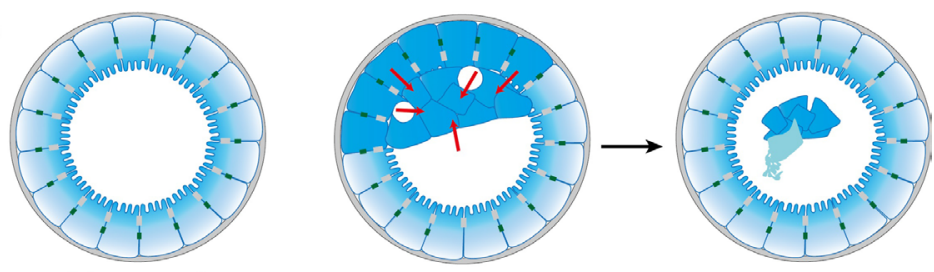

Normal

C
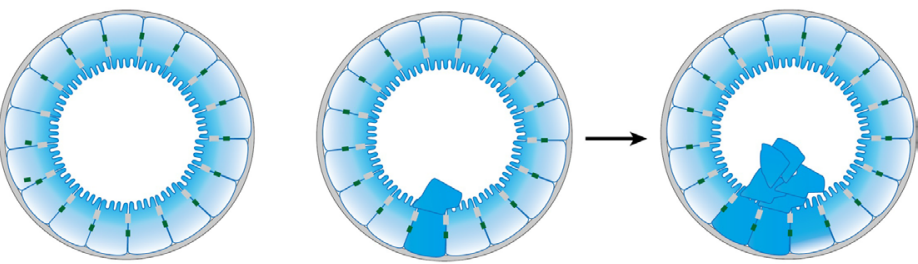

Normal

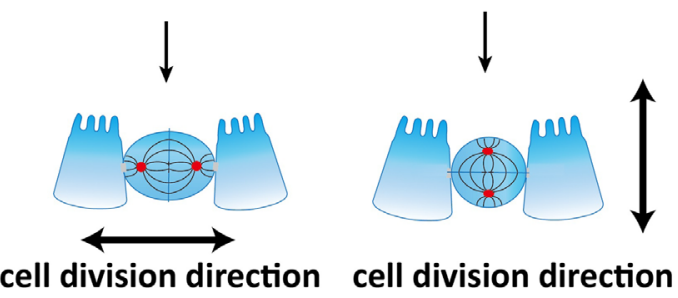

Figure 6: Polarized epithelial tissue architecture suppresses tumorigenesis through three hypothesized mechanisms. (A) Epithelial tissue architecture as a whole serves as a non-cell-autonomous tumor suppressor by pushing mutant cells into the lumen. The mutant cell is shown in red and will undergo apoptosis, as illustrated by the development of filled-lumen cysts shown in Figure 2G. (B) Epithelial tissue architecture as a whole serves as a non-cell-autonomous tumor suppressor by releasing abnormal cells into the lumen through lumen-merge. A multi-lumen cyst (e.g., the cyst shown in the last image of Figure 2B) could evolve to a filled-lumen cyst (e.g., the cyst pointed by white arrow in Figure 2B) by merging the lumens and releasing the often multi-layered and not apicobasal-polarized cell mass (indicated by red arrows) into the lumen. The cell mass will eventually undergo apoptosis. (C) Spindle orientation maintains epithelial monolayer and suppresses tumorigenesis. Misorientated spindles lead to cells dividing into the lumen, forming polyps (see the cyst pointed by a white arrow in Figure 2C). 
GTP-bound Rab11 [28-30] and functions as a GEF toward Rab39 [15, 31]. AVL9 contains DENN-domain related AH regions and is reported to function in the late secretory pathway in yeast [16]. Notably, AVL9-depletion inhibits the migration of A549 human adenocarcinomic alveolar basal epithelial cells [17] (in our study, AVL9-depletion promotes MDCKII cell migration; the difference may be due to different pathways in which AVL9 participates between MDCKII and A549 cells). AVL9 has been proposed to be a GEF for Rab11 or Rab4, although such GEF activity has not been experimentally detected [17].

AVL9- and DENND5A-knockdown clones in our study share many abnormalities, which may be explained by their common interaction with Rab11 [15, 17, 32] as discussed above. Primarily associated with the pericentriolar recycling endosomes, the Rab11 subfamily members (Rab11a, Rab11b, and Rab25) are critical players in intracellular membrane trafficking [33, 34]. Significantly, a Rab11a-directed network governs de novo generation of apical membrane and lumen in MDCK cells $[14,35,36]$. During the first cell division, apical membrane polarity complex proteins such as Crumbs3a and CDC42 are concentrated in Rab11a-positive recycling endosomes, partitioned between two daughter cells before cytokinesis, and delivered to the site of cytokinesis [14, 36]. This generates a first apical membrane that eventually forms the lumen. Thus, because DENND5A and likely AVL9 interact with Rab11 [15, 17], it is possible that knockdown of either gene perturbs this Rab11a-directed network, resulting in defective lumen establishment during the first cell division. Indeed, the abnormal phenotypes observed during the lumen-establishing stage of AVL9- or $D E N N D 5 A$-knockdown clones match those of Rab11adepleted cells [14].

Rab11-dependent membrane trafficking is also required for the completion of cytokinesis [37, 38], and Rab11 has been shown to regulate spindle alignment by modulating metaphase microtubule dynamics in $C$. elegans early embryos [39]. As hypothesized above, these functions are consistent with cytokinesis defects and spindle misalignment observed in AVL9- or DENND5Aknockdown clones.

Besides the similarities described above, the two gene-knockdowns also differ. First, DENND5Aknockdown clones have developed more small-sized cysts. This may be explained by DENND5A's additional interactions with Rab6, which regulates vesicular trafficking within the Golgi and post-Golgi compartments $[32,40,41]$. Importantly, Rab6A' (one of the isoforms) has been proposed to regulate the metaphase/anaphase transition by inactivating Mad2-spindle checkpoint through its regulators/effectors [42], and Rab6A' alteration blocks cells in metaphase [43]. Thus, it is possible that DENND5A-knockdown alters the function of Rab6A', blocking the cells in metaphase which leads to the development of multinucleated cells and small-sized cysts.
Significantly, DENND5A-knockdown yields cysts with cells actively growing into the lumen, resulting in a phenotype resembling human colonic polyps. This observation supports the tumor suppressing role of DENND5A and is consistent with other DENN domaincontaining proteins such as DENND2D, which is proposed to be a tumor suppressor of gastric cancer [44]. How the polyp-like phenotype develops in DENND5Aknockdown cells needs further studies. Is it because of spindle misorientation alone or in combination with other mechanisms that make the cells reenter the cell cycle? Lastly, the protein expression of DENND5A has increased sharply in certain abnormal and apoptotic cells. The underlying mechanism and the significance of this finding require further studies.

Clearly, more studies are required to understand the molecular mechanisms through which DENND5A and $A V L 9$ contribute to MDCKII cystogenesis. To investigate the relationship between each gene and the Rab GTPases discussed above may yield valuable insights into the mechanisms.

\section{Our study supports the notion that epithelial polarity is a tumor suppressor}

Epithelia such as those from the colon are highly organized tissues consisting of a single layer of cells with apicobasal polarity. While loss of cell polarity and break down of tissue architecture are hallmarks of epithelial cancers, whether they are drivers or passengers of cancer is however less clear [4-11]. Our work reported here supports that they are cancer drivers, as polarized epithelial tissue architecture itself functions as a non-cellautonomous tumor suppressor as hypothesized below.

First, those filled-lumen cysts developed in the knockdown clones nicely illustrate that epithelial tissue architecture as whole can serve as a non-cell-autonomous tumor suppressor, as previously hypothesized [5, 6]. The knockdown of each gene results in cell cycle defects, yielding cells carrying additional abnormal changes. Many such cells are then being ejected to the lumen, possibly through mechanisms such as "purse string" wound-healing [6], and are disintegrated through apoptosis (Figure 6A). Eliminating these cells better maintains the integrity of the cyst and allows the cyst to grow. Indeed, the filled-lumen cysts in AVL9-knockdown cells become increasing more normal-looking (e.g., in apicobasal polarity) at later stages of cystogenesis. It is possible that mutant cells are also eliminated similarly (Figure 6A) in the colon and rectum, suppressing colorectal tumorigenesis.

Some filled-lumen cysts of AVL9-knockdown clones harbor live cells. We hypothesize that these cysts are actually evolved from multi-lumen cysts that have emerged earlier. As a multi-lumen cyst develops, the lumens enlarge and merge at certain points, pushing the often multi-layered and not apicobasal-polarized cell 
mass into the lumen (Figure 6B). The cell mass is then no longer attached to any matrix and will eventually undergo apoptosis. Thus, this process can also eliminate abnormal cells from the system (Figure 6B), which in our hypothesis provides another mechanism for polarized epithelial architecture functioning as a tumor suppressor.

Finally, correctly orientated spindles maintain the epithelial monolayer [22], directly suppressing tumorigenesis (Figure 6C). To the contrary, misorientated spindles lead to cells dividing into the lumen, forming polyps (Figure 6C). This is possibly a mechanism for the development of the polyp-like phenotype in DENND5Aknockdown cells.

More studies are clearly required to test these hypotheses (Figure 6) and to understand the molecular pathways through which polarized epithelial tissue architecture suppresses tumorigenesis in a non-cellautonomous fashion. The importance of non-cellautonomous regulations in cancer has been elegantly illustrated by a recent publication [45].

\section{METHODS}

\section{MDCKII cell culture}

MDCKII cells, kindly provided by Dr. Karl Matlin of University of Chicago, were grown in Eagle's minimal essential medium (MEM) with 5\% (v/v) fetal bovine serum (FBS), $100 \mathrm{U} / \mathrm{ml}$ penicillin, $100 \mathrm{mg} / \mathrm{ml}$ streptomycin, $10 \mathrm{mM}$ HEPES and $2 \mathrm{mM}$ Glutamine. For $3 \mathrm{D}$ culture, MDCKII cells were grown in matrigel, either embedded or on-top, to develop cysts as described [14].

\section{Antibodies and fluorescent dyes}

Antibodies used include anti-E-cadherin (Santa Cruz, Dallas, Texas, USA), anti- $\alpha$-tubulin (DM1A clone from Sigma, Saint Louis, MO, USA), anti- $\gamma$-tubulin (Abcam, Cambridge, MA), anti-cleaved caspase3 (Cell Signaling, Danvers, MA, USA), anti-NUPL1 (Sigma), anti-DENND5A (Abcam), and anti-AVL9 (Abcam). Alexa Fluor $^{\circledR} 488$ or 647 conjugated secondary antibodies are from Jackson Immunoresearch, West Grove, PA, USA. Rhodamine phalloidin for F-actin-staining and DAPI (1:1000) for nucleus-staining are from Invitrogen, Grand Island, NY, USA.

\section{Immunofluorescence and confocal microscopy}

MDCKII cysts from 3D cell culture were immunostained by indirect immunofluorescence as described [46]. Briefly, the cysts in matrix were fixed with $4 \%$ PFA, permeablized with permeablization solution ( $1 \times$
PBS with $0.5 \%$ Triton X-100) and blocked with blocking solution (1x PBS with 10\% FBS, 3\% bovine serum albumin, $0.2 \%$ Triton X-100, and $0.2 \%$ Tween-20). The cysts were then hybridized with primary antibodies at $4{ }^{\circ} \mathrm{C}$ overnight followed by secondary antibodies (and DAPI for nuclear staining and rhodamine phalloidin for F-actin staining when needed) at room temperature for 2-3 hours. Finally, the mounting solution, Fluoro-Gel from Electron Microscopy Sciences, was added, and images were taken with a Zeiss LSM 710 confocal microscope.

\section{shRNA plasmid constructs}

shRNA plasmids were constructed using the pRNAi-H1-puro vector system (Biosettia, San Diego, CA, USA) per the manufacturer's instruction. Briefly, the oligonucleotide encoding shRNA sequences (see Supplementary Table S1) were cloned into the pRNAi-H1Puro vector, which contains the H1 RNA polymerase III promoter and the puromycin marker. The cloned plasmids were confirmed by Sanger sequencing. The plasmid containing oligonucleotide encoding shRNA against the lacZ gene was provided by the company (Biosettia, San Diego, CA, USA) and used as the control.

\section{Nucleofection and selection of stable knockdown clones}

The shRNA plasmids were linearized by $\mathrm{PacI}$ and introduced into the MDCKII cells by nucleofection using the Amaxa Cell Line Nucleofector Kit L (Lonza, Allendale, NJ, USA), following the manufacturer's instructions. Transfected cells were selected by growing in medium containing $4 \mu \mathrm{g} / \mathrm{mL}$ puromycin until $>99 \%$ cells in the mock-infected wells were killed. Single colonies were then picked and expanded. At the same time, RNA from the subculture of the cells was extracted and the knockdown efficiency was evaluated by qRT-PCR. The stable knockdown clones were maintained in the presence of $4 \mu \mathrm{g} / \mathrm{mL}$ puromycin.

\section{Migration assay}

The scratch-wound cell migration assay was carried out as described [17]. Briefly, more than $1 \times 10^{5}$ of control and knockdown cells were plated in triplicate in 24-well plates. Twenty four hours later after reaching $>90 \%$ confluency, the growth media were replaced with those without FBS. Twelve hours later, wounds were scratched in the monolayer using a $200 \mu \mathrm{l}$ pipette tip, and pictures were taken at different time points after the scratch. 


\section{ACKNOWLEDGEMENTS}

We thank Dr. Karl Matlin of the University of Chicago for providing us the MDCKII cells and technical help in growing these cells; Drs. Gan Fu and Jinmei Shen for their contributions to the study; Dr. Muthugapatti K. Kandasamy for help in our imaging work; Dr. Michael J McEachern for help in cell migration assays; Dr. Stephen Hajduk for help in cell transfection; and Dr. Youxi Luo for help in statistic analysis. Imaging was performed in the Biomedical Microscopy Core at the University of Georgia. The work is supported by the NCI R01 CA182093, American Cancer Society, Georgia Cancer Coalition, and the AKC Canine Health Foundation.

\section{References}

1. Tang J, Li Y, Lyon K, Camps J, Dalton S, Ried T and Zhao S. Cancer driver-passenger distinction via sporadic human and dog cancer comparison: a proof-of-principle study with colorectal cancer. Oncogene. 2013.

2. Tang J, Le S, Sun L, Yan X, Zhang M, Macleod J, Leroy B, Northrup N, Ellis A, Yeatman TJ, Liang Y, Zwick ME and Zhao S. Copy number abnormalities in sporadic canine colorectal cancers. Genome research. 2010; 20(3):341-350.

3. Youmans L, Taylor C, Shin E, Harrell A, Ellis AE, Seguin $\mathrm{B}, \mathrm{Ji} \mathrm{X}$ and Zhao S. Frequent alteration of the tumor suppressor gene APC in sporadic canine colorectal tumors. PloS one. 2012; 7(12):e50813.

4. Royer C and Lu X. Epithelial cell polarity: a major gatekeeper against cancer? Cell death and differentiation. 2011; 18(9):1470-1477.

5. Bissell MJ and Hines WC. Why don't we get more cancer? A proposed role of the microenvironment in restraining cancer progression. Nature medicine. 2011; 17(3):320-329.

6. Lee M and Vasioukhin V. Cell polarity and cancer--cell and tissue polarity as a non-canonical tumor suppressor. Journal of cell science. 2008; 121(Pt 8):1141-1150.

7. Wodarz A and Nathke I. Cell polarity in development and cancer. Nature cell biology. 2007; 9(9):1016-1024.

8. Igaki T, Pagliarini RA and Xu T. Loss of cell polarity drives tumor growth and invasion through $\mathrm{JNK}$ activation in Drosophila. Current biology : CB. 2006; 16(11):1139-1146.

9. Krishnan M, Lapierre LA, Knowles BC and Goldenring JR. Rab25 regulates integrin expression in polarized colonic epithelial cells. Molecular biology of the cell. 2013; 24(6):818-831.

10. Feigin ME, Akshinthala SD, Araki K, Rosenberg AZ, Muthuswamy LB, Martin B, Lehmann BD, Berman HK, Pietenpol JA, Cardiff RD and Muthuswamy SK. Mislocalization of the Cell Polarity Protein Scribble Promotes Mammary Tumorigenesis and Is Associated with Basal Breast Cancer. Cancer research. 2014; 74(11):31803194.
11. Polyak K and Hu M. Do myoepithelial cells hold the key for breast tumor progression? Journal of mammary gland biology and neoplasia. 2005; 10(3):231-247.

12. D'Angelo MA and Hetzer MW. Structure, dynamics and function of nuclear pore complexes. Trends in cell biology. 2008; 18(10):456-466.

13. Schwartz SL, Cao C, Pylypenko O, Rak A and WandingerNess A. Rab GTPases at a glance. Journal of cell science. 2007; 120(Pt 22):3905-3910.

14. Bryant DM, Datta A, Rodriguez-Fraticelli AE, Peranen J, Martin-Belmonte F and Mostov KE. A molecular network for de novo generation of the apical surface and lumen. Nature cell biology. 2010; 12(11):1035-1045.

15. Marat AL, Dokainish $\mathrm{H}$ and McPherson PS. DENN domain proteins: regulators of Rab GTPases. The Journal of biological chemistry. 2011; 286(16):13791-13800.

16. Harsay E and Schekman R. Av19p, a member of a novel protein superfamily, functions in the late secretory pathway. Molecular biology of the cell. 2007; 18(4):1203-1219.

17. Linford A, Yoshimura S, Nunes Bastos R, Langemeyer L, Gerondopoulos A, Rigden DJ and Barr FA. Rab14 and its exchange factor FAM116 link endocytic recycling and adherens junction stability in migrating cells. Developmental cell. 2012; 22(5):952-966.

18. Dukes JD, Whitley P and Chalmers AD. The MDCK variety pack: choosing the right strain. BMC cell biology. 2011; 12:43.

19. Elia N and Lippincott-Schwartz J. Culturing MDCK cells in three dimensions for analyzing intracellular dynamics. Current protocols in cell biology / editorial board, Juan S Bonifacino [et al]. 2009; Chapter 4:Unit 422.

20. Datta A, Bryant DM and Mostov KE. Molecular regulation of lumen morphogenesis. Current biology : CB. 2011; 21(3):R126-136.

21. Weigelt B, Ghajar CM and Bissell MJ. The need for complex 3D culture models to unravel novel pathways and identify accurate biomarkers in breast cancer. Adv Drug Deliver Rev. 2014; 69:42-51.

22. Jaffe AB, Kaji N, Durgan J and Hall A. Cdc42 controls spindle orientation to position the apical surface during epithelial morphogenesis. The Journal of cell biology. 2008; 183(4):625-633.

23. Guan TL, Muller S, Klier G, Pante N, Blevitt JM, Haner M, Paschal B, Aebi U and Gerace L. Structural-Analysis of the P62 Complex, an Assembly of O-Linked Glycoproteins That Localizes near the Central Gated Channel of the Nuclear-Pore Complex. Molecular biology of the cell. 1995; 6(11):1591-1603.

24. Tillement V, Remy MH, Raynaud-Messina B, Mazzolini L, Haren L and Merdes A. Spindle assembly defects leading to the formation of a monopolar mitotic apparatus. Biology of the cell / under the auspices of the European Cell Biology Organization. 2009; 101(1):1-11.

25. Blagosklonny MV. Mitotic arrest and cell fate: why and 
how mitotic inhibition of transcription drives mutually exclusive events. Cell cycle. 2007; 6(1):70-74.

26. Chatel G and Fahrenkrog B. Nucleoporins: leaving the nuclear pore complex for a successful mitosis. Cellular signalling. 2011; 23(10):1555-1562.

27. Cross MK and Powers MA. Nup98 regulates bipolar spindle assembly through association with microtubules and opposition of MCAK. Molecular biology of the cell. 2011; 22(5):661-672.

28. Fukuda M, Kobayashi H, Ishibashi K and Ohbayashi N. Genome-wide investigation of the Rab binding activity of RUN domains: development of a novel tool that specifically traps GTP-Rab35. Cell structure and function. 2011; 36(2):155-170.

29. Fernandes H, Franklin E and Khan AR. Crystallization of an engineered RUN domain of Rab6-interacting protein 1/DENND5. Acta crystallographica Section F, Structural biology and crystallization communications. 2011; 67(Pt 5):556-560.

30. Recacha R, Boulet A, Jollivet F, Monier S, Houdusse A, Goud B and Khan AR. Structural basis for recruitment of Rab6-interacting protein 1 to Golgi via a RUN domain. Structure. 2009; 17(1):21-30.

31. Yoshimura S, Gerondopoulos A, Linford A, Rigden DJ and Barr FA. Family-wide characterization of the DENN domain Rab GDP-GTP exchange factors. The Journal of cell biology. 2010; 191(2):367-381.

32. Miserey-Lenkei S, Waharte F, Boulet A, Cuif MH, Tenza D, El Marjou A, Raposo G, Salamero J, Heliot L, Goud B and Monier S. Rab6-interacting protein 1 links Rab6 and Rab11 function. Traffic. 2007; 8(10):1385-1403.

33. Kelly EE, Horgan CP and McCaffrey MW. Rab11 proteins in health and disease. Biochemical Society transactions. 2012; 40(6):1360-1367.

34. Hales CM, Griner R, Hobdy-Henderson KC, Dorn MC, Hardy D, Kumar R, Navarre J, Chan EK, Lapierre LA and Goldenring JR. Identification and characterization of a family of Rab11-interacting proteins. The Journal of biological chemistry. 2001; 276(42):39067-39075.

35. Desclozeaux M, Venturato J, Wylie FG, Kay JG, Joseph SR, Le HT and Stow JL. Active Rab11 and functional recycling endosome are required for E-cadherin trafficking and lumen formation during epithelial morphogenesis. American journal of physiology Cell physiology. 2008; 295(2):C545556.

36. Schluter MA, Pfarr CS, Pieczynski J, Whiteman EL, Hurd TW, Fan S, Liu CJ and Margolis B. Trafficking of Crumbs 3 during cytokinesis is crucial for lumen formation. Molecular biology of the cell. 2009; 20(22):4652-4663.

37. Lee Y, Chung S, Baek IK, Lee TH, Paik SY and Lee J. UNC119a bridges the transmission of Fyn signals to Rab11, leading to the completion of cytokinesis. Cell cycle. 2013; 12(8):1303-1315.

38. Yu X, Prekeris R and Gould GW. Role of endosomal Rab
GTPases in cytokinesis. European journal of cell biology. 2007; 86(1):25-35.

39. Zhang H, Squirrell JM and White JG. RAB-11 permissively regulates spindle alignment by modulating metaphase microtubule dynamics in Caenorhabditis elegans early embryos. Molecular biology of the cell. 2008; 19(6):25532565.

40. Grigoriev I, Splinter D, Keijzer N, Wulf PS, Demmers J, Ohtsuka T, Modesti M, Maly IV, Grosveld F, Hoogenraad $\mathrm{CC}$ and Akhmanova A. Rab6 regulates transport and targeting of exocytotic carriers. Developmental cell. 2007; 13(2):305-314.

41. Martinez O, Schmidt A, Salamero J, Hoflack B, Roa M and Goud B. The small GTP-binding protein rab6 functions in intra-Golgi transport. The Journal of cell biology. 1994; 127(6 Pt 1):1575-1588.

42. Cuif MH, Possmayer F, Zander H, Bordes N, Jollivet F, Couedel-Courteille A, Janoueix-Lerosey I, Langsley G, Bornens M and Goud B. Characterization of GAPCenA, a GTPase activating protein for Rab6, part of which associates with the centrosome. The EMBO journal. 1999; 18(7):1772-1782.

43. Miserey-Lenkei S, Couedel-Courteille A, Del Nery E, Bardin S, Piel M, Racine V, Sibarita JB, Perez F, Bornens $\mathrm{M}$ and Goud B. A role for the Rab6A' GTPase in the inactivation of the Mad2-spindle checkpoint. The EMBO journal. 2006; 25(2):278-289.

44. Kanda M, Shimizu D, Nomoto S, Takami H, Hibino S, Oya H, Hashimoto R, Suenaga M, Inokawa Y, Kobayashi D, Tanaka C, Yamada S, Fujii T, Nakayama G, Sugimoto $\mathrm{H}$, Koike M, et al. Prognostic impact of expression and methylation status of DENN/MADD domain-containing protein 2D in gastric cancer. Gastric cancer : official journal of the International Gastric Cancer Association and the Japanese Gastric Cancer Association. 2014.

45. Marusyk A, Tabassum DP, Altrock PM, Almendro V, Michor F and Polyak K. Non-cell-autonomous driving of tumour growth supports sub-clonal heterogeneity. Nature. 2014; 514(7520):54-58.

46. Ling C, Zuo D, Xue B, Muthuswamy S and Muller WJ. A novel role for 14-3-3sigma in regulating epithelial cell polarity. Genes \& development. 2010; 24(9):947-956. 